\title{
The Decarboxylative Blaise Reaction
}

Jae Hoon Lee, Bo Seung Choi, Jay Hyok Chang, Hee Bong Lee, Joo-Yong Yoon, Jaeick Lee, and Hyunik Shin*

Chemical Development Division, LG Life Sciences, Ltd./R\&D, 104-

1, Moonji-dong, Yusong-gu, Daejeon 305-380, Korea.

hisin@1gls.com

\section{Contents}

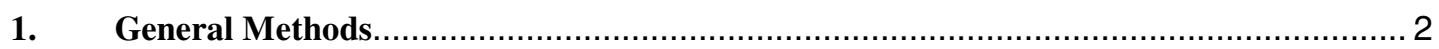

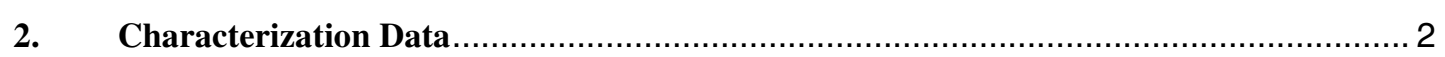

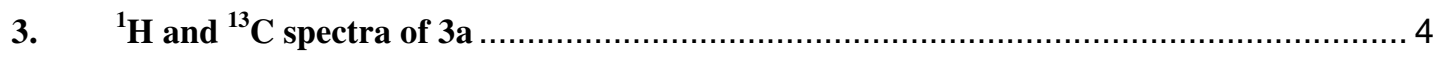

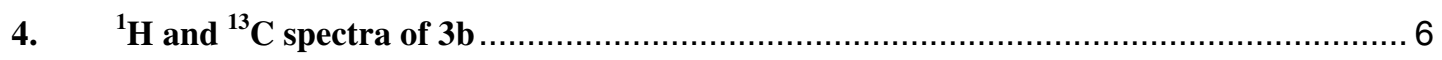

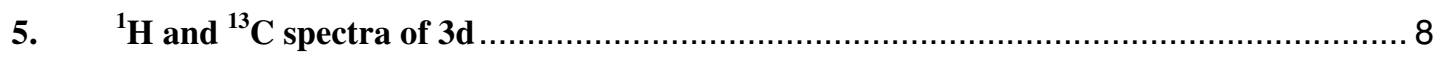

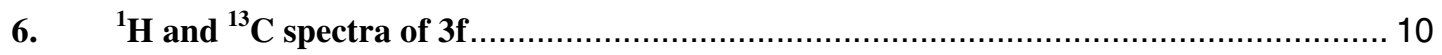

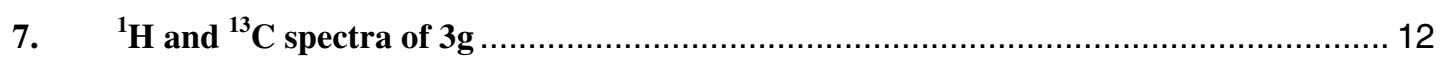

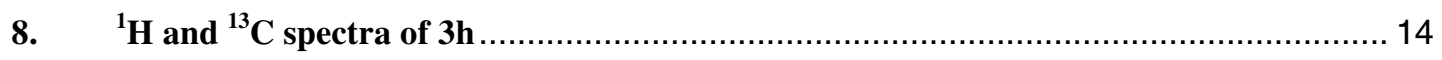

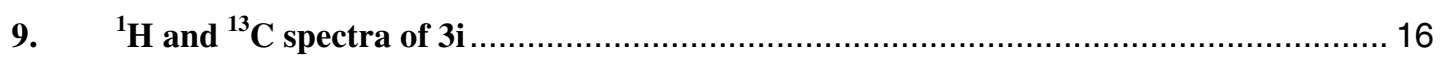

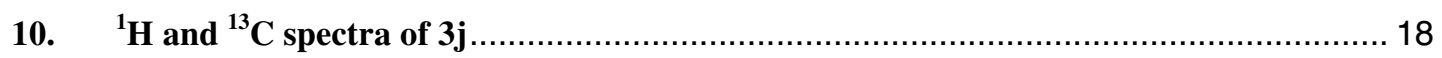

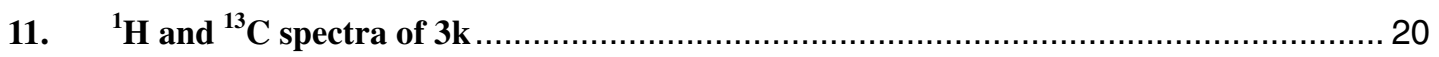




\section{General Methods}

All reactions were performed under nitrogen atmosphere. All commercially available reagents and solvents were used without further purification unless otherwise noted. Column chromatography was performed with silica gel $(0.063-0.2 \mathrm{~mm})$. The combined organic layers were dried over MgSO4. Solvents were evaporated under reduced pressure. All yields given refer to as isolated yields. NMR spectra were recorded on a $400 \mathrm{MHz}$ spectrometer. Chemical shifts (delta) are reported in ppm downfield from tetramethylsilane. Coupling constants (J values) are reported in Hertz. HRMS experiments were performed on a hybrid quadrupole time-of-flight mass spectrometer. LC-MS experiments were performed using a single-quadrupole mass spectrometer equipped with HPLC system.

\section{Characterization Data}

(Z)-3-Amino-3-phenyl-acrylic acid ethyl ester (3a): ${ }^{1}{ }^{1} \mathrm{H}$ MNR (400 MHz, $\left.\mathrm{CDCl}_{3}\right): \delta$ 7.45-7.38 (m, 2 H), 7.33-7.24 (m, $3 \mathrm{H}), 4.85$ (s, $1 \mathrm{H}), 4.05$ (q, $J=7.1 \mathrm{~Hz}, 2 \mathrm{H}), 1.18$ (t, $J=7.1 \mathrm{~Hz}, 3 \mathrm{H}) ;{ }^{13} \mathrm{C}$ NMR $(100$ $\left.\mathrm{MHz}, \mathrm{CDCl}_{3}\right) \delta 170.4,160.5,137.72,130.2,128.8,126.1,84.5,58.9,14.6$; LC-MS (ESI) m/z: 192.2 $(\mathrm{M}+1)$.

(Z)-3-Amino-3-(4-bromo-phenyl)-acrylic acid ethyl ester (3b): ${ }^{1} \mathrm{H} \mathrm{MNR}\left(400 \mathrm{MHz}, \mathrm{CDCl}_{3}\right) \delta 7.54$ (d, $J=8.5 \mathrm{~Hz}, 2 \mathrm{H}), 7.41$ (d, $J=8.5 \mathrm{~Hz}, 2 \mathrm{H}), 4.93(\mathrm{~s}, 1 \mathrm{H}), 4.16(\mathrm{q}, J=7.1 \mathrm{~Hz}, 2 \mathrm{H}), 1.29$ (t, $J=7.1 \mathrm{~Hz}, 3$ $\mathrm{H}) ;{ }^{13} \mathrm{C}$ NMR (100 MHz, $\left.\mathrm{CDCl}_{3}\right) \delta 170.1,159.1,136.6,132.0$, 127.7, 124.4, 85.1, 50.0, 14.6; LC-MS (ESI) m/z: $270.0(\mathrm{M}+1), 272.1(\mathrm{M}+1)$; HRMS (ESI) Calcd for $\mathrm{C}_{11} \mathrm{H}_{13} \mathrm{BrNO}_{2}: 270.0124$. Found 270.0123 $\left(\mathrm{M}+\mathrm{H}^{+}\right)$.

(Z)-3-Amino-3-p-tolyl-acrylic acid ethyl ester (3d): ${ }^{2}{ }^{1} \mathrm{H}$ MNR (400 MHz, $\left.\mathrm{CDCl}_{3}\right) \delta 7.50(\mathrm{~m}, 2 \mathrm{H}), 6.92$ (m, $2 \mathrm{H}), 4.93$ (s, $1 \mathrm{H}), 4.16$ (q, $J=7.1 \mathrm{~Hz}, 2 \mathrm{H}), 3.83$ (s, $3 \mathrm{H}), 1.29$ (t, $J=7.1 \mathrm{~Hz}, 3 \mathrm{H}) ;{ }^{13} \mathrm{C}$ NMR $(100$ $\left.\mathrm{MHz}, \mathrm{CDCl}_{3}\right) \delta 170.4,161.1,160.1,129.7,127.4,114.0,83.5,58.7,55.3,14.5$; LC-MS (ESI) m/z: 206.2 $(\mathrm{M}+1)$.

(Z)-3-Amino-3-(4-methoxy-phenyl)-acrylic acid ethyl ester (3f): ${ }^{1} \mathrm{H} \mathrm{MNR}\left(400 \mathrm{MHz}, \mathrm{CDCl}_{3}\right) \delta 7.42$. (d, $J=7.9 \mathrm{~Hz}, 2 \mathrm{H}), 7.19$ (d, $J=7.9 \mathrm{~Hz}, 2 \mathrm{H}), 4.94$ (s, $1 \mathrm{H}$ ), 4.16 (q, $J=7.1 \mathrm{~Hz}, 2 \mathrm{H}$ ), 2.36 (s, $3 \mathrm{H}), 1.29$ (t, $J=7.1 \mathrm{~Hz}, 3 \mathrm{H}) ;{ }^{13} \mathrm{C} \mathrm{NMR}\left(100 \mathrm{MHz}, \mathrm{CDCl}_{3}\right) \delta 170.3,160.4,140.3,134.5,129.3,125.9,83.8,58.7$, 21.1, 14.4; LC-MS (ESI) m/z: $222.1\left(\mathrm{M}+1\right.$ ); HRMS (ESI) Calcd for $\mathrm{C}_{12} \mathrm{H}_{16} \mathrm{NO}_{3}$ : 222.1124. Found $222.1122\left(\mathrm{M}+\mathrm{H}^{+}\right)$.

(Z)-3-Amino-3-pyridin-2-yl-acrylic acid ethyl ester (3i): ${ }^{1} \mathrm{H} \mathrm{MNR}\left(400 \mathrm{MHz}, \mathrm{CDCl}_{3}\right) \delta 8.62(\mathrm{~d}, 1 \mathrm{H})$, 7.74 (m, $2 \mathrm{H}), 7.34$ (m, $1 \mathrm{H}), 5.34$ (s, $1 \mathrm{H}), 4.20$ (q, $J=7.1 \mathrm{~Hz}, 2 \mathrm{H}), 1.31$ (t, $J=7.1 \mathrm{~Hz}, 3 \mathrm{H}) ;{ }^{13} \mathrm{C}$ NMR $\left(100 \mathrm{MHz}, \mathrm{CDCl}_{3}\right) \delta 170.5,155.4,151.2,148.6,136.7,124.6,120.1,82.2,58.9,14.5 ;$ LC-MS (ESI) m/z: $193.1(\mathrm{M}+1)$, $194.1(\mathrm{M}+2)$; HRMS (ESI) Calcd for $\mathrm{C}_{10} \mathrm{H}_{13} \mathrm{~N}_{2} \mathrm{O}_{2}$ : 193.0971. Found $193.0977\left(\mathrm{M}+\mathrm{H}^{+}\right)$.

\footnotetext{
${ }^{1}$ Bagley, M.C.; Brace, C.; Dale, J.W.; Ohnesorge, M.; Phillips, N. G.; Xiong, X.; Bower, J. J. Chem. Soc. Perkin Trans 1 2002, 1663.

2 Litinas, K. E.; Nicolaides, D. N.; Varella, E. A. J. Heterocyclic Chem. 1990, 27, 769.
} 
(Z)-3-Amino-3-furan-3-yl-acrylic acid ethyl ester (3j): ${ }^{3} \mathrm{H} \mathrm{MNR}\left(400 \mathrm{MHz}, \mathrm{CDCl}_{3}\right) \delta 7.73(\mathrm{~m}, 1 \mathrm{H})$, 7.44 (m, $1 \mathrm{H}), 6.55$ (m, $1 \mathrm{H}), 4.94(\mathrm{~s}, 1 \mathrm{H}), 4.15$ (q, $J=7.1 \mathrm{~Hz}, 2 \mathrm{H}), 1.29(\mathrm{t}, J=7.1 \mathrm{~Hz}, 3 \mathrm{H}) ;{ }^{13} \mathrm{C} \mathrm{NMR}$ $\left(100 \mathrm{MHz}, \mathrm{CDCl}_{3}\right) \delta 170.3,152.3,143.9,140.6,124.0,107.9,83.7,58.8,14.5$; LC-MS (ESI) m/z:182.1 $(\mathrm{M}+1)$; HRMS (ESI) Calcd for $\mathrm{C}_{9} \mathrm{H}_{12} \mathrm{NO}_{3}: 182.0811$. Found $182.0815\left(\mathrm{M}+\mathrm{H}^{+}\right)$.

(Z)-3-Amino-3-cyclopropyl-acrylic acid ethyl ester (3k): ${ }^{1} \mathrm{H} \mathrm{MNR}\left(400 \mathrm{MHz}, \mathrm{CDCl}_{3}\right) \delta 4.43(\mathrm{~s}, 1 \mathrm{H})$, 4.06 (q, $J=7.1 \mathrm{~Hz}, 2 \mathrm{H}), 1.39$ (m, $1 \mathrm{H}), 1.23$ (t, $J=7.1 \mathrm{~Hz}, 3 \mathrm{H}), 0.84(\mathrm{~m}, 2 \mathrm{H}), 0.71(\mathrm{~m}, 2 \mathrm{H}) ;{ }^{13} \mathrm{C} \mathrm{NMR}$ $\left(100 \mathrm{MHz}, \mathrm{CDCl}_{3}\right) \delta 170.3,165.1,80.5,58.4,15.7,14.5,7.0$; LC-MS (ESI) m/z: 156.1 (M+1); HRMS (ESI) Calcd for $\mathrm{C}_{8} \mathrm{H}_{14} \mathrm{NO}_{2}: 156.1019$. Found $156.1020\left(\mathrm{M}+\mathrm{H}^{+}\right)$.

${ }^{3}$ Rantohul, Y. K.; Chartrand, A. Org. Lett. 2007, 9, 1029. 
3. ${ }^{1} \mathrm{H}$ and ${ }^{13} \mathrm{C}$ spectra of $3 \mathrm{a}$

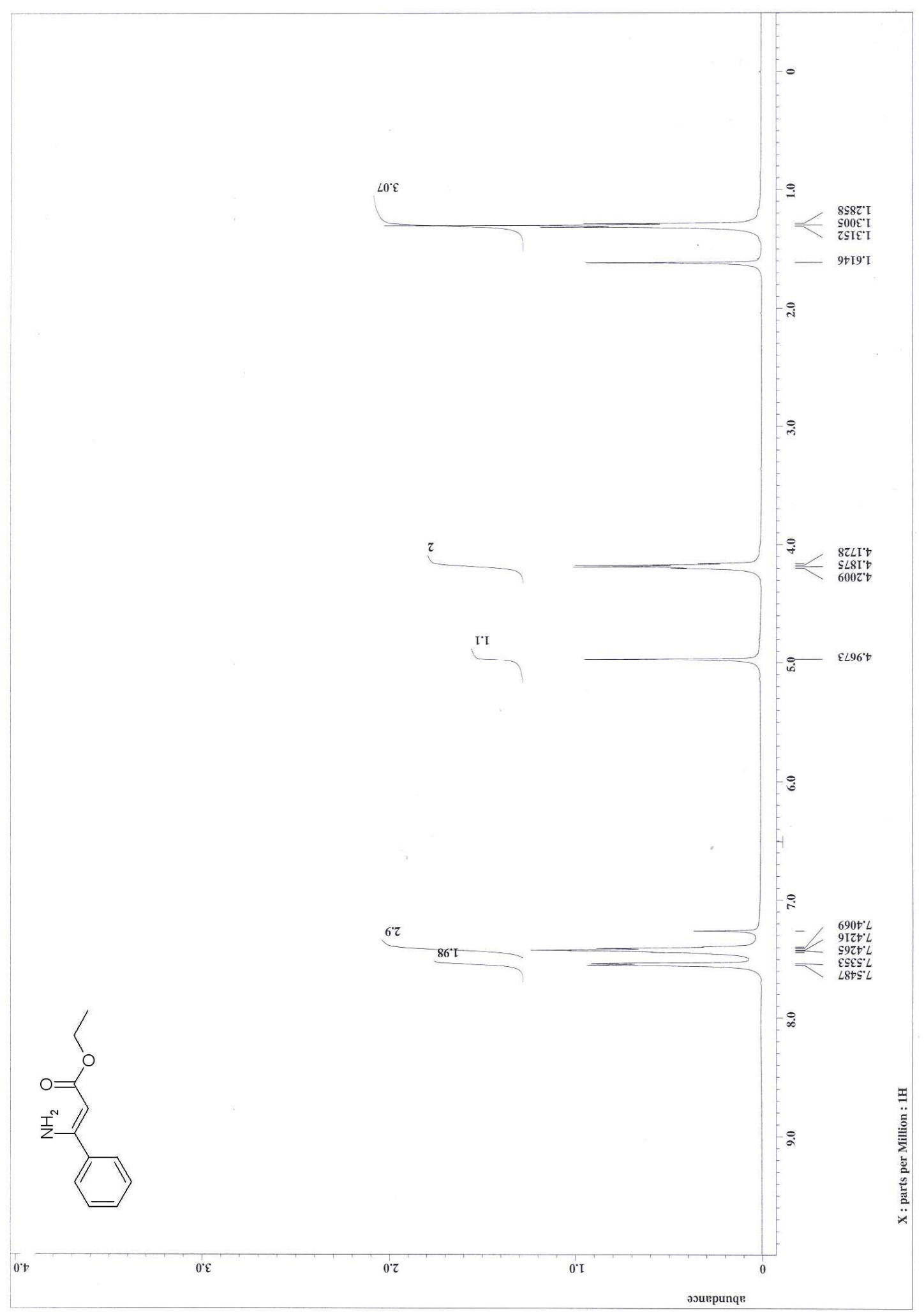




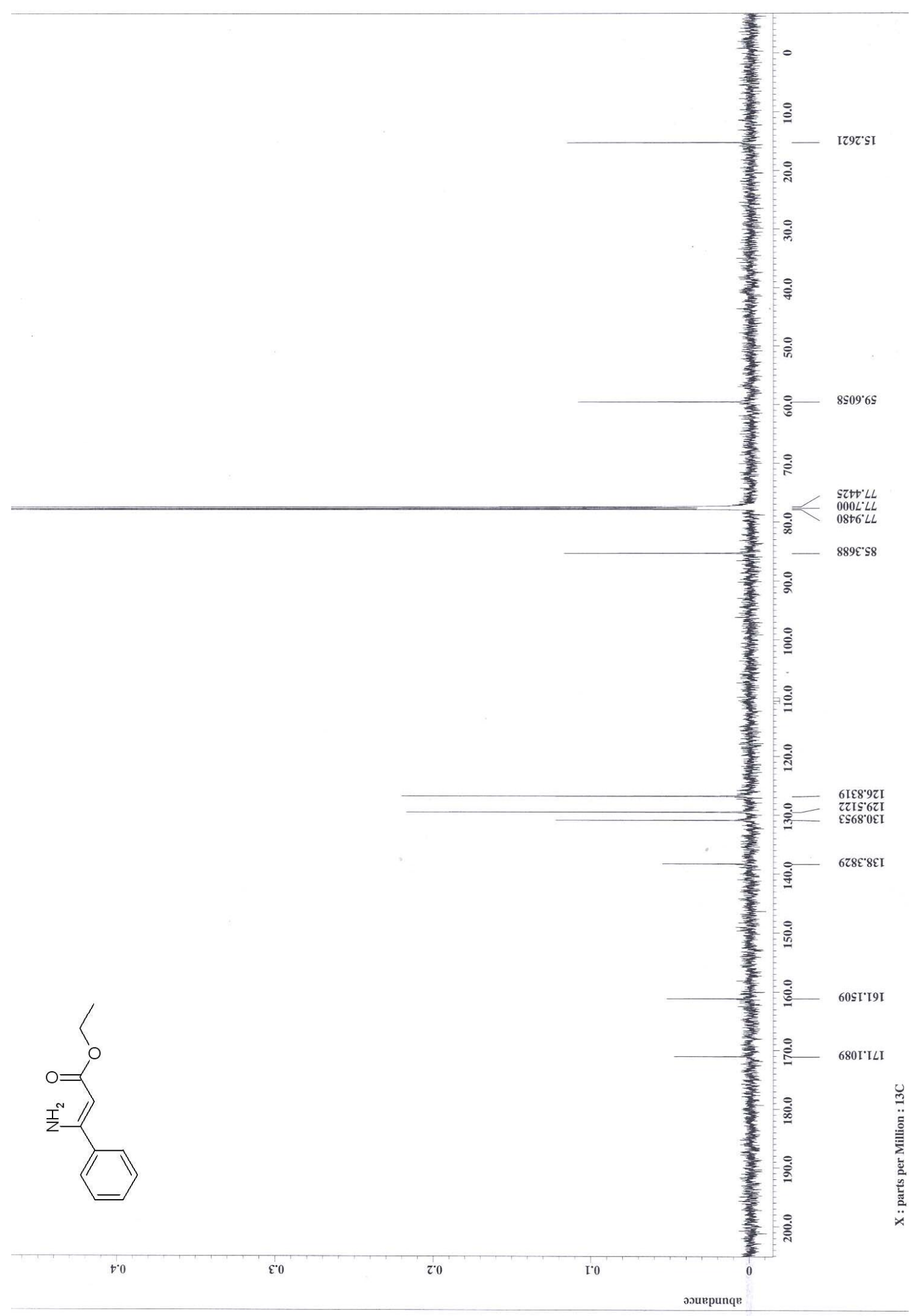


4. ${ }^{1} \mathrm{H}$ and ${ }^{13} \mathrm{C}$ spectra of $3 \mathrm{~b}$

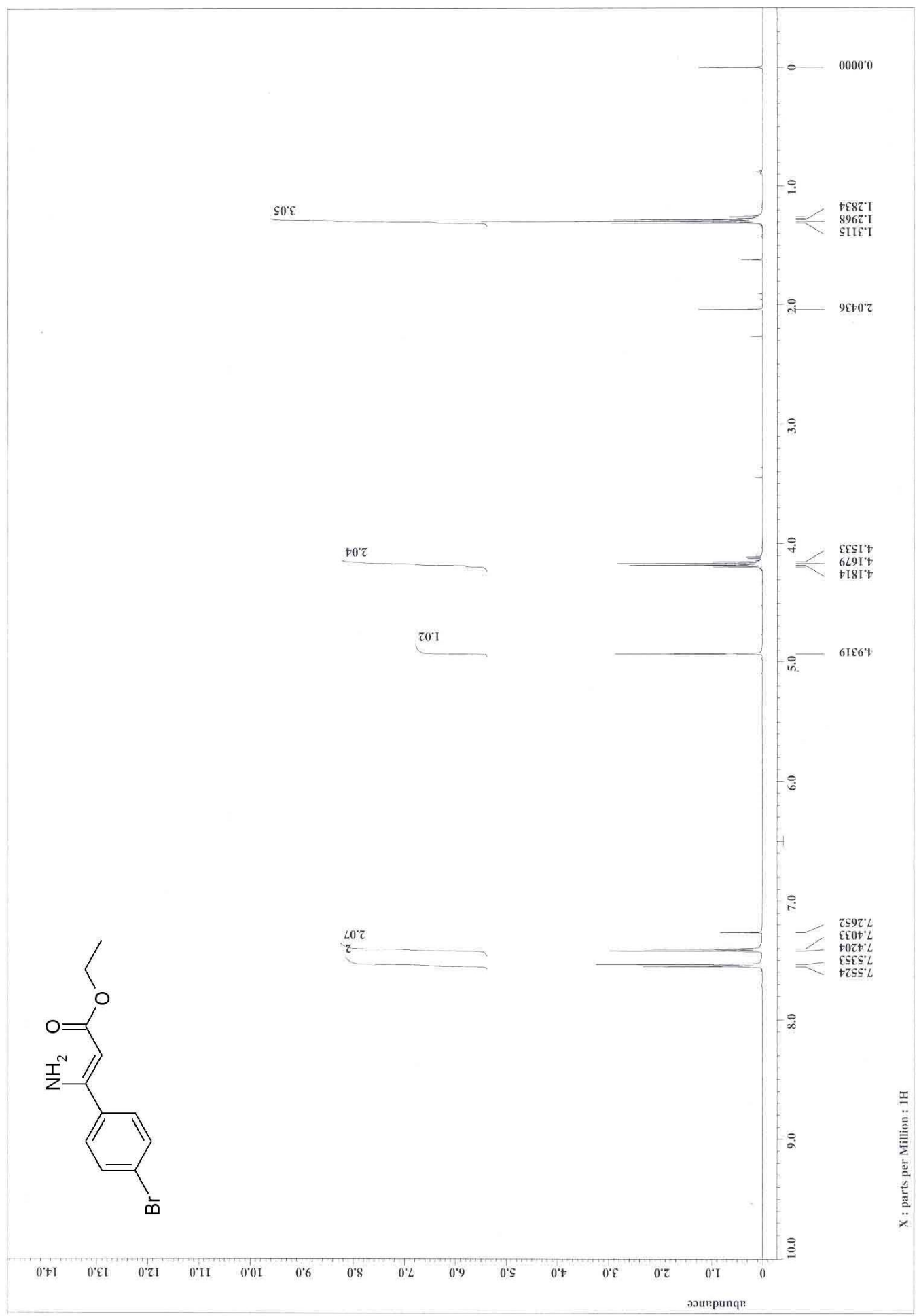




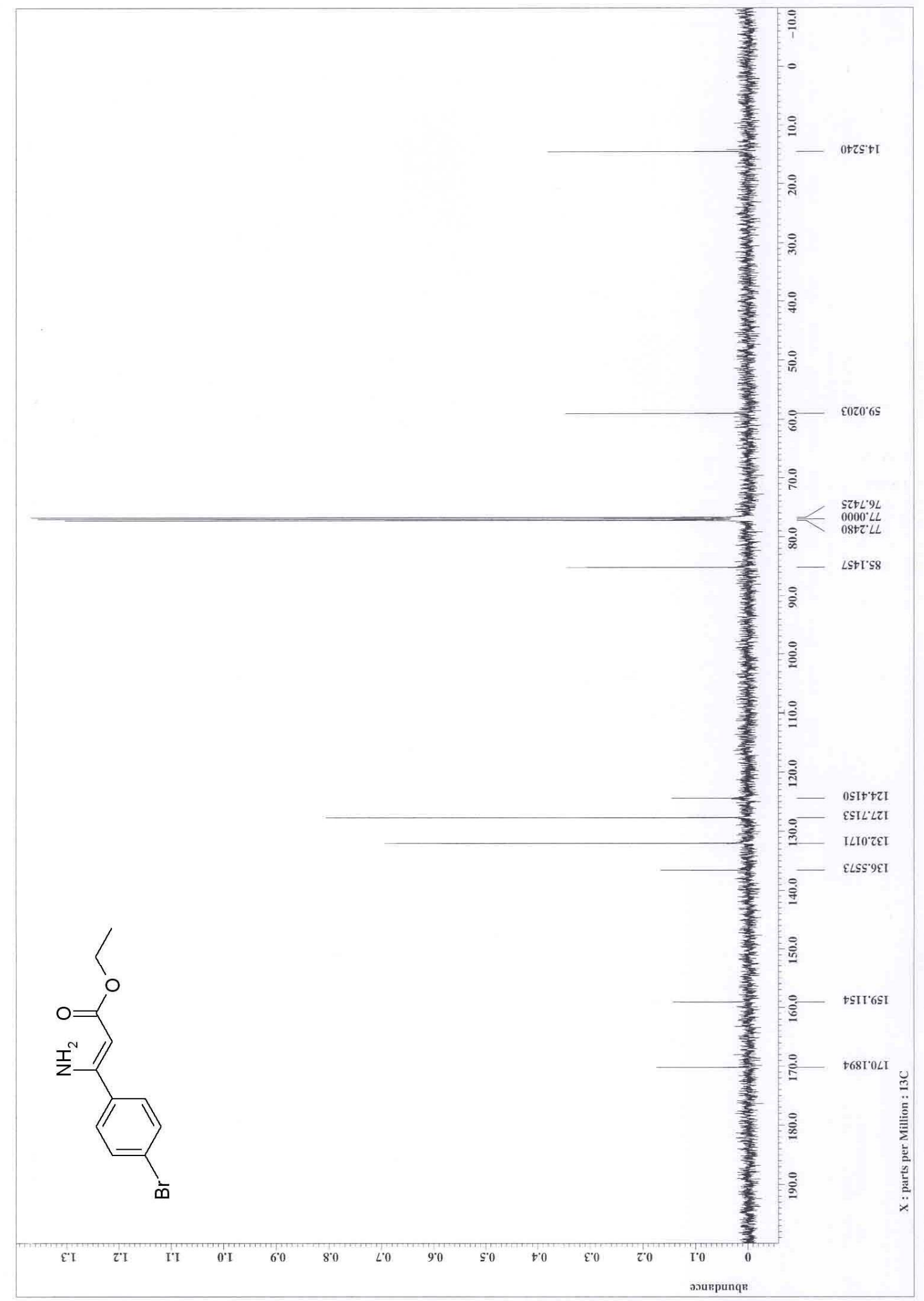


5. ${ }^{1} \mathrm{H}$ and ${ }^{13} \mathrm{C}$ spectra of $3 \mathrm{~d}$

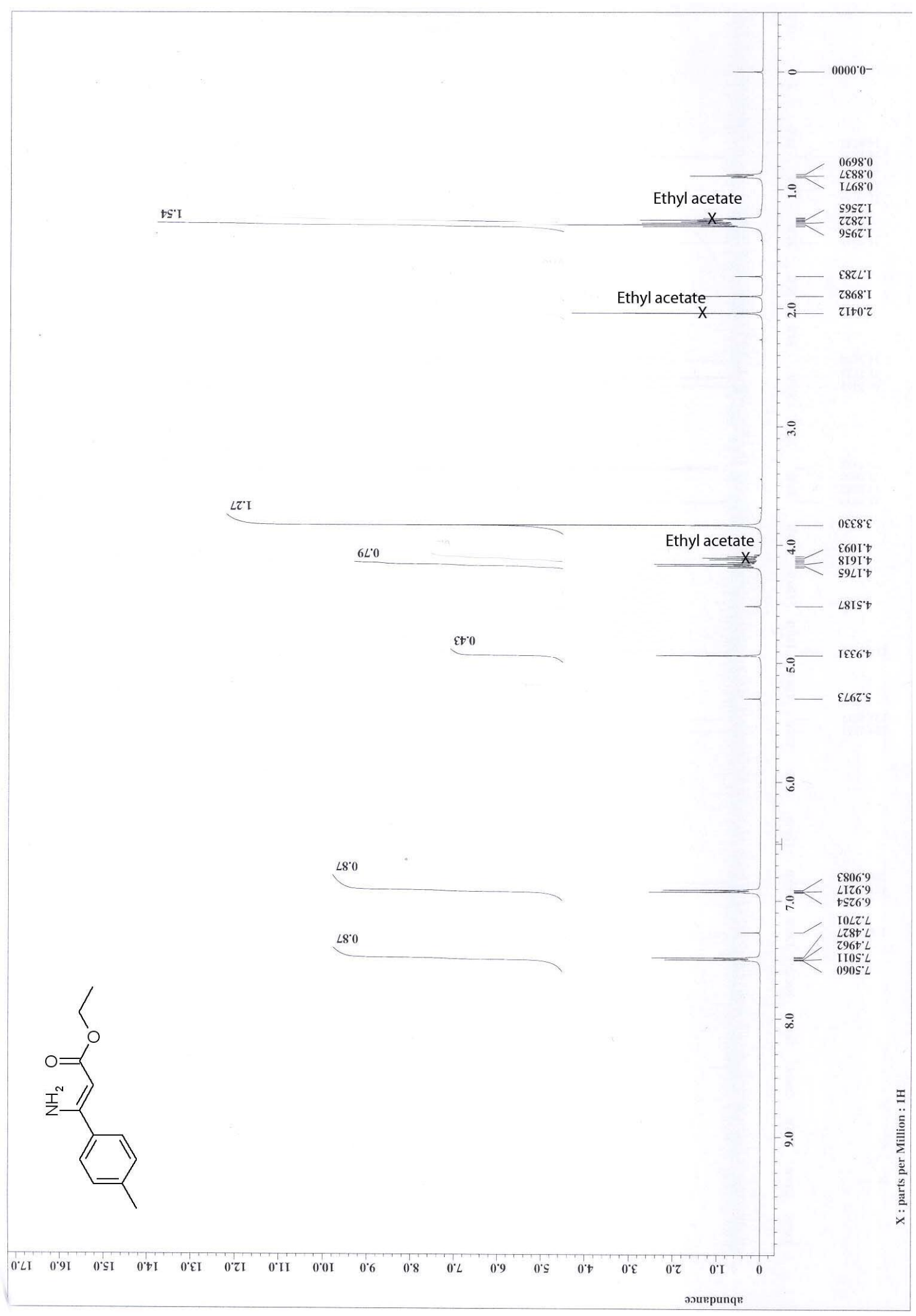




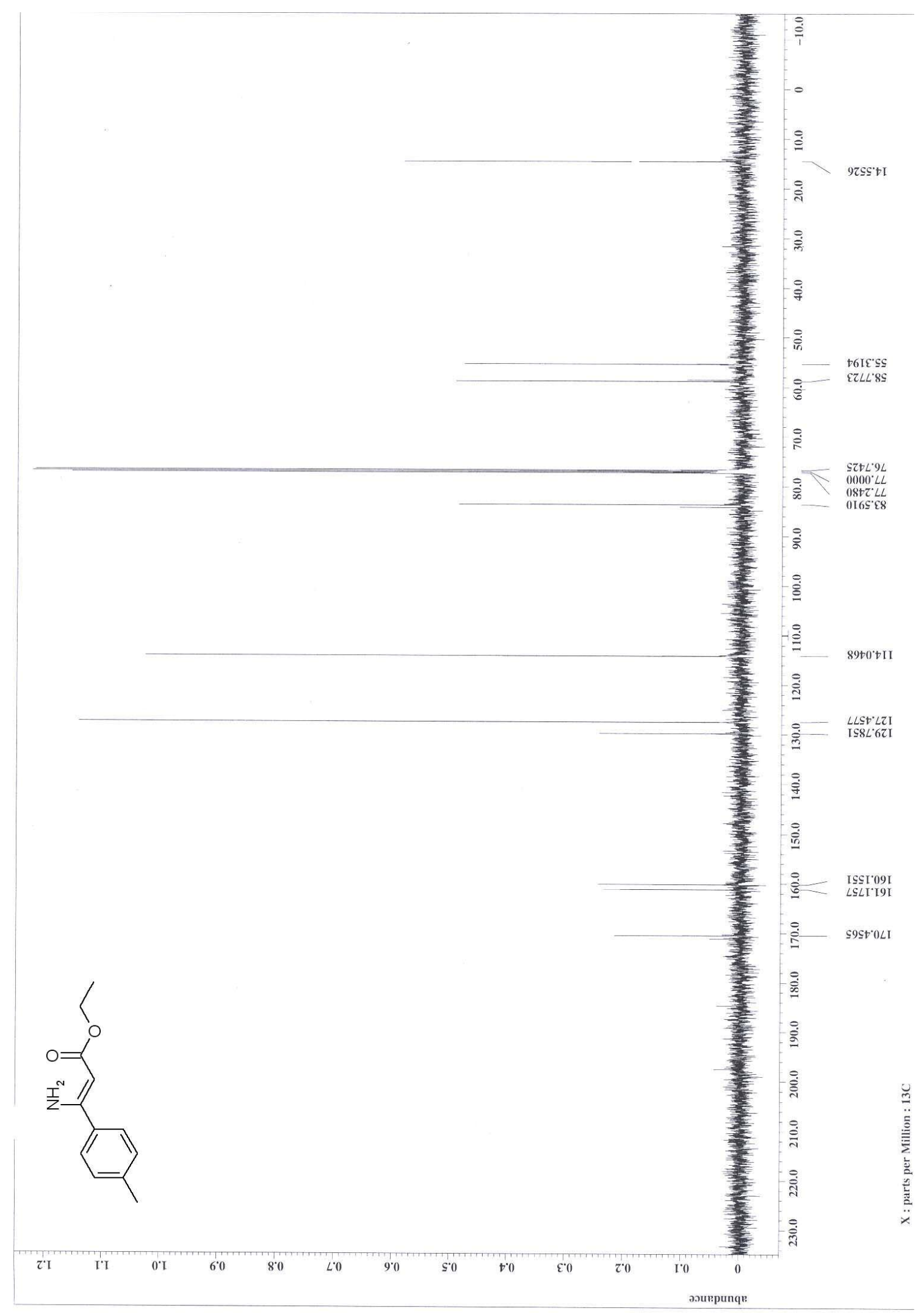


6. ${ }^{1} \mathrm{H}$ and ${ }^{13} \mathrm{C}$ spectra of $3 \mathrm{f}$

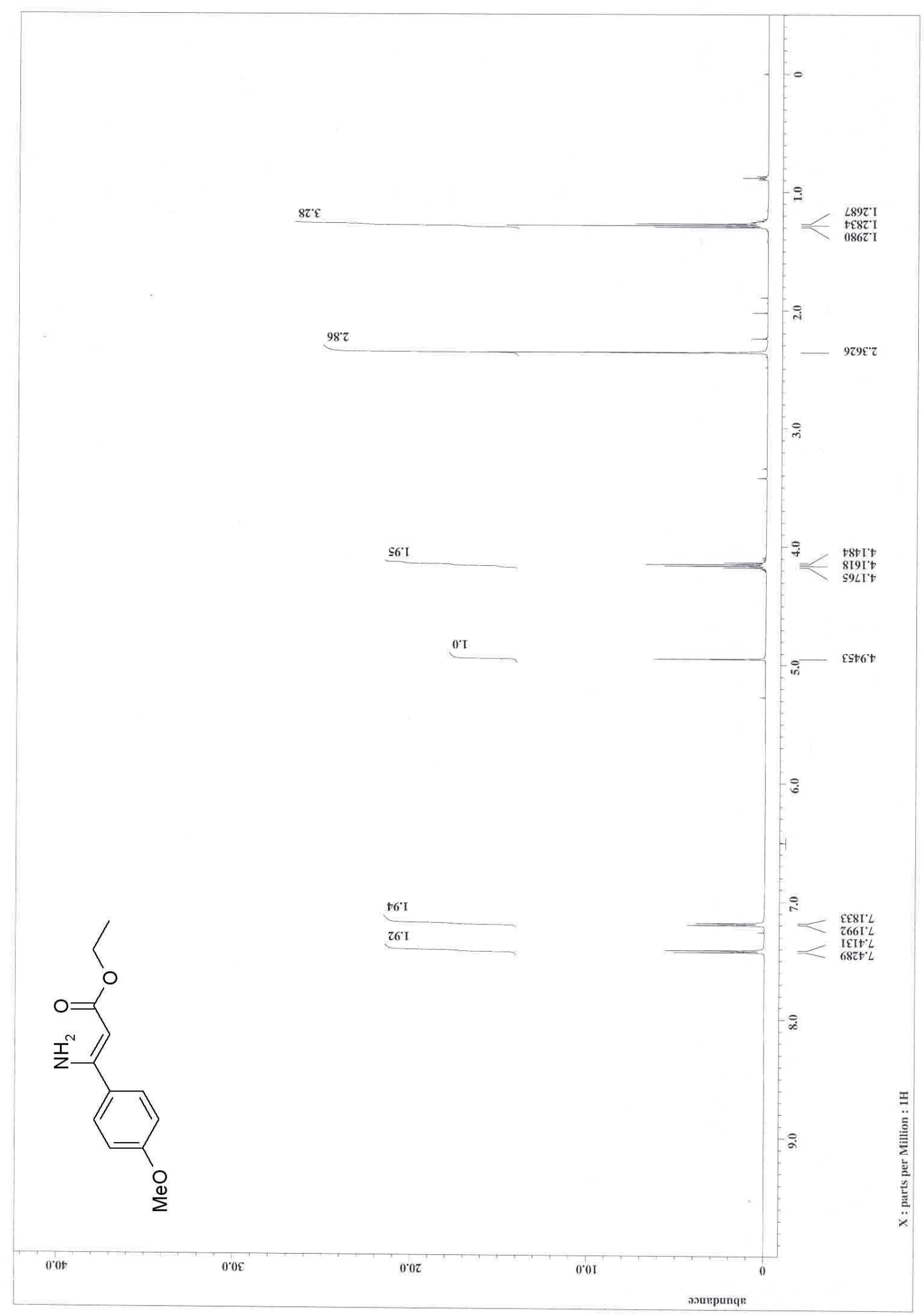




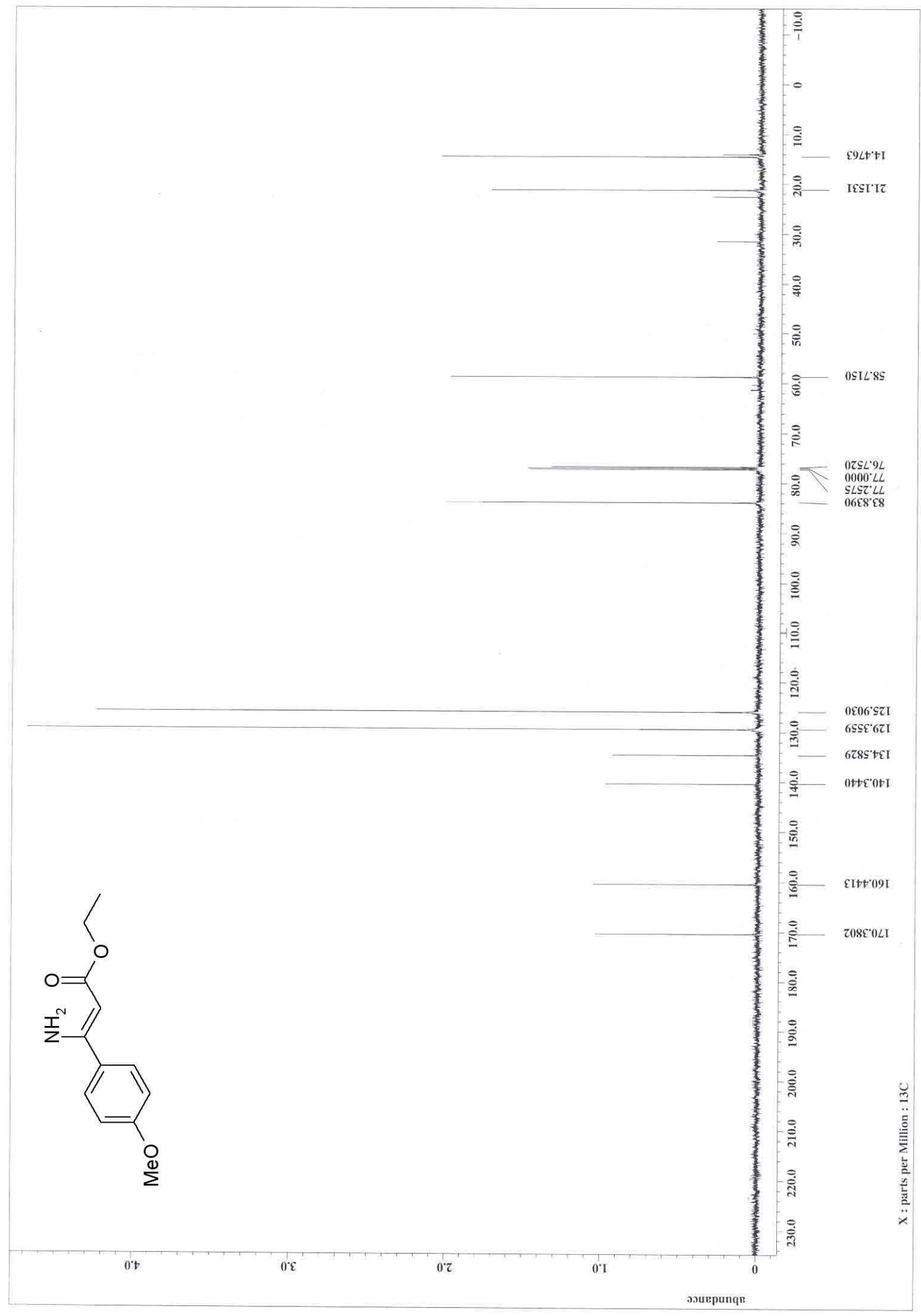


7. ${ }^{1} \mathrm{H}$ and ${ }^{13} \mathrm{C}$ spectra of $3 \mathrm{~g}$

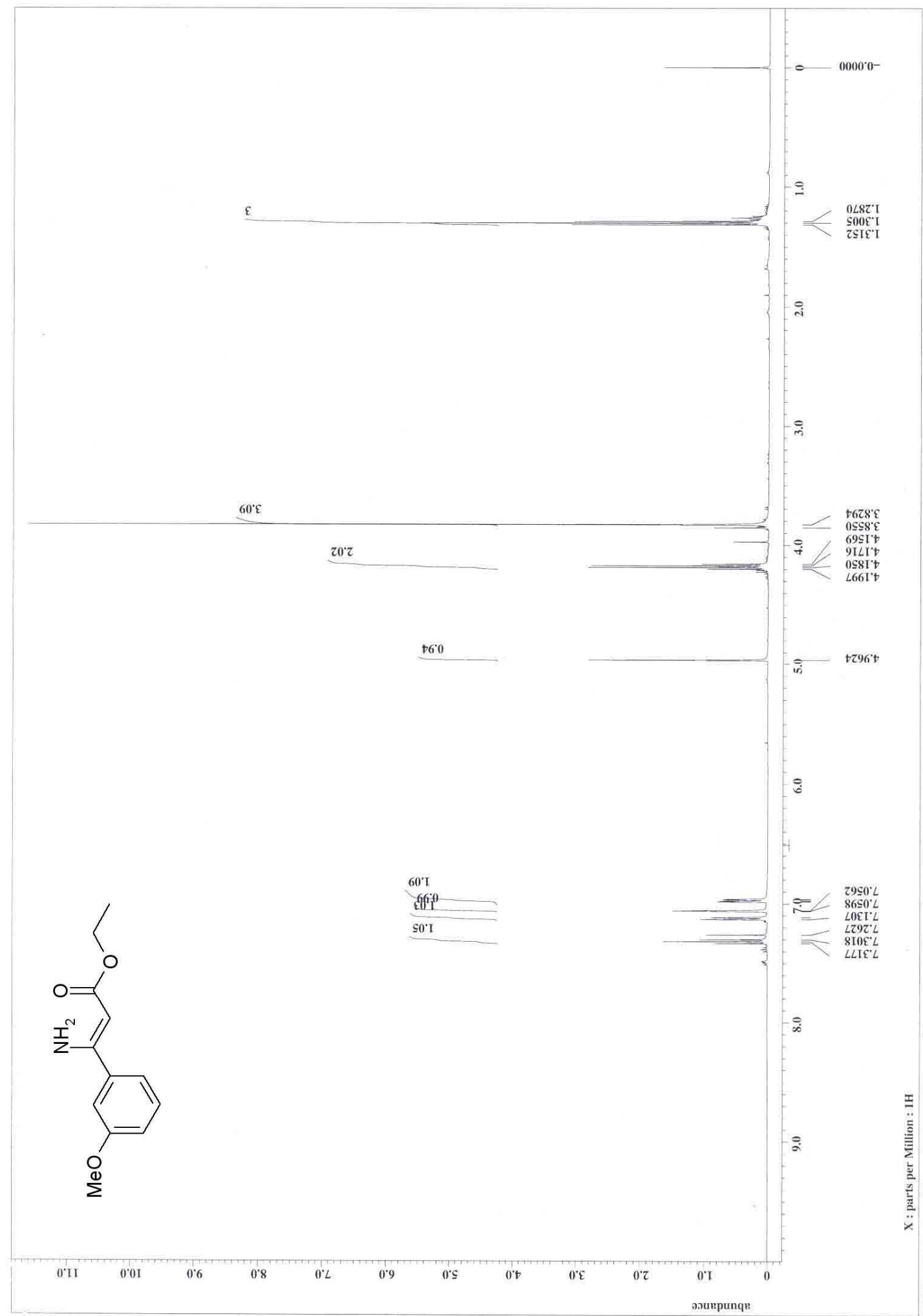




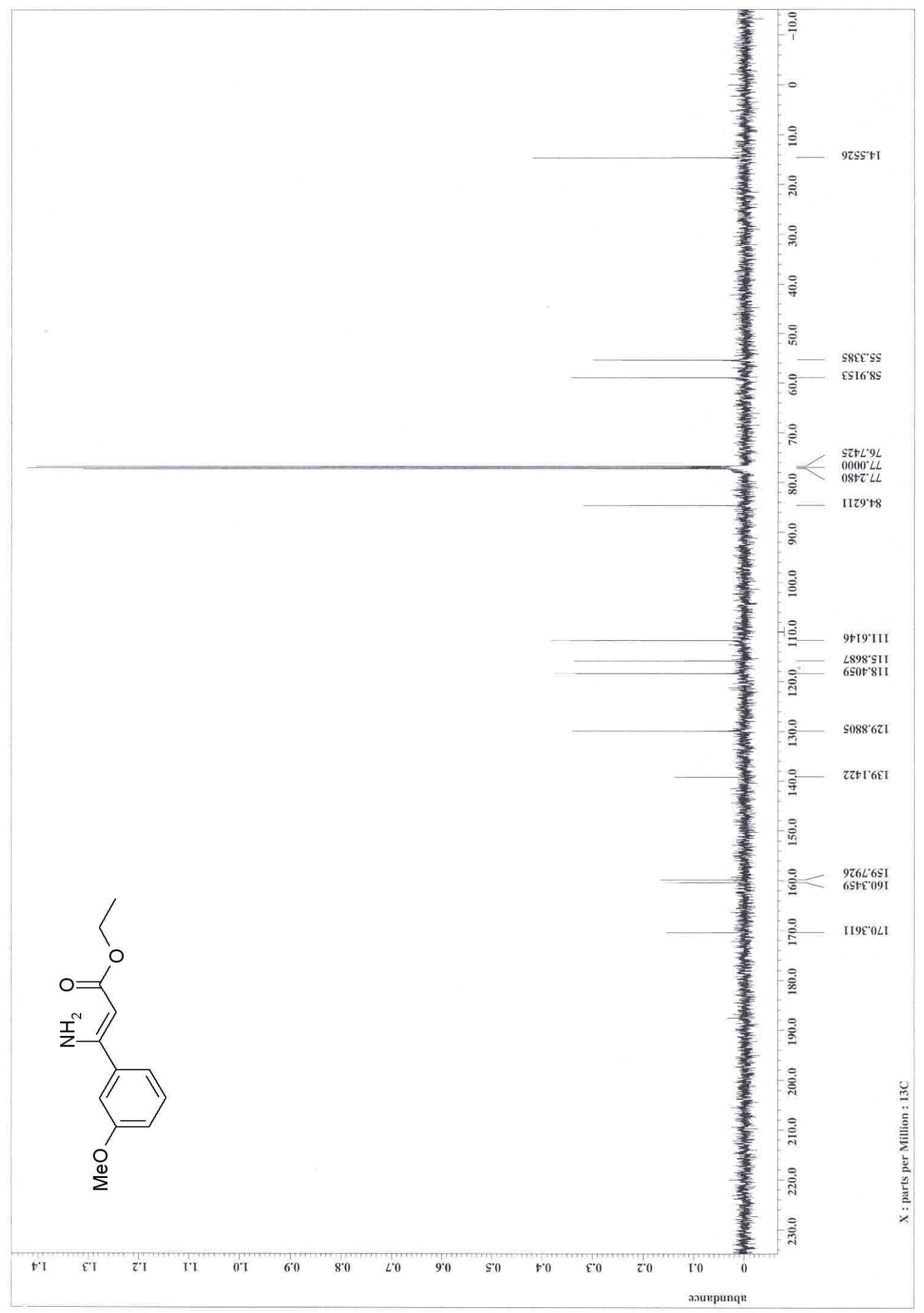


8. ${ }^{1} \mathrm{H}$ and ${ }^{13} \mathrm{C}$ spectra of $3 \mathrm{~h}$

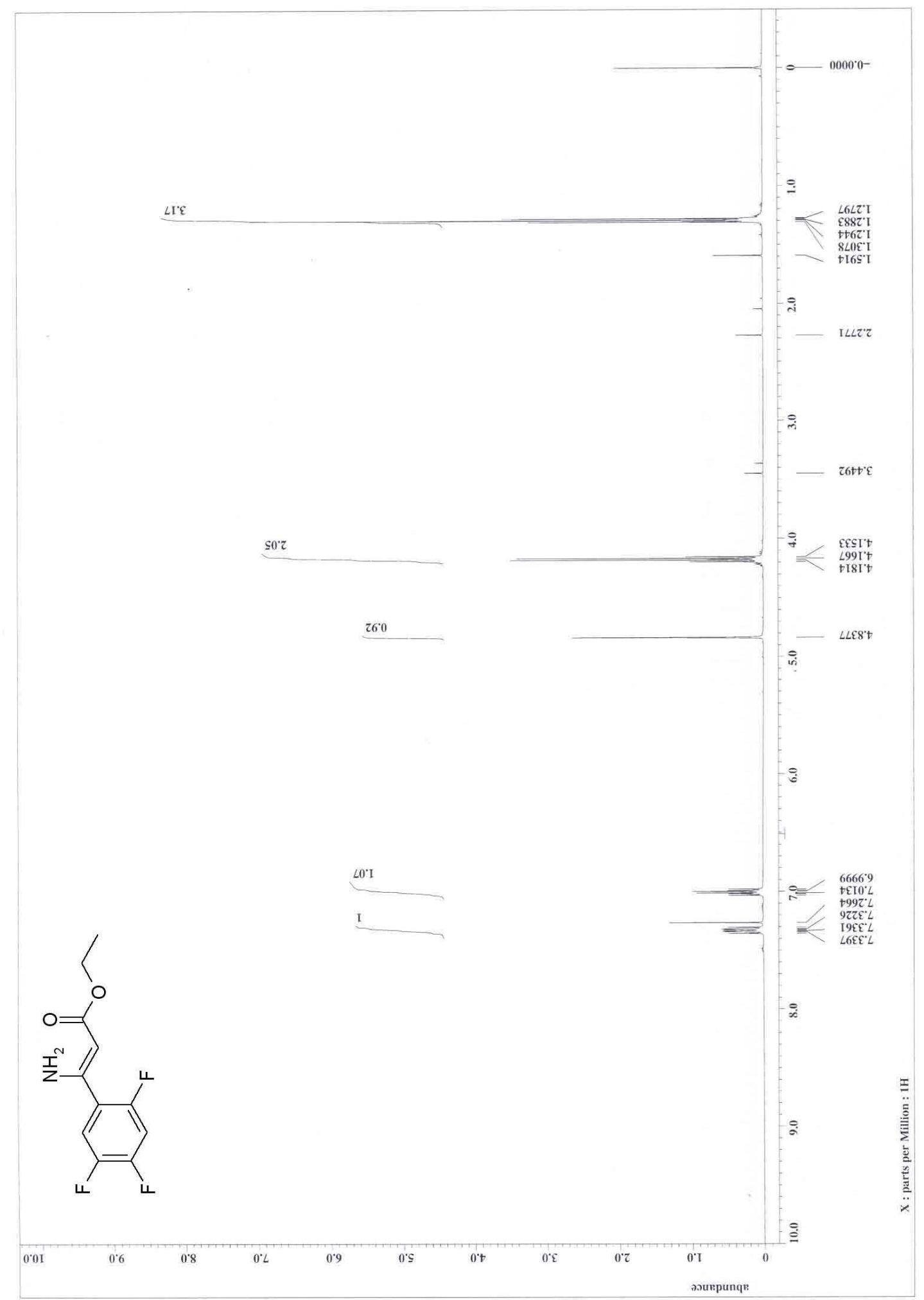



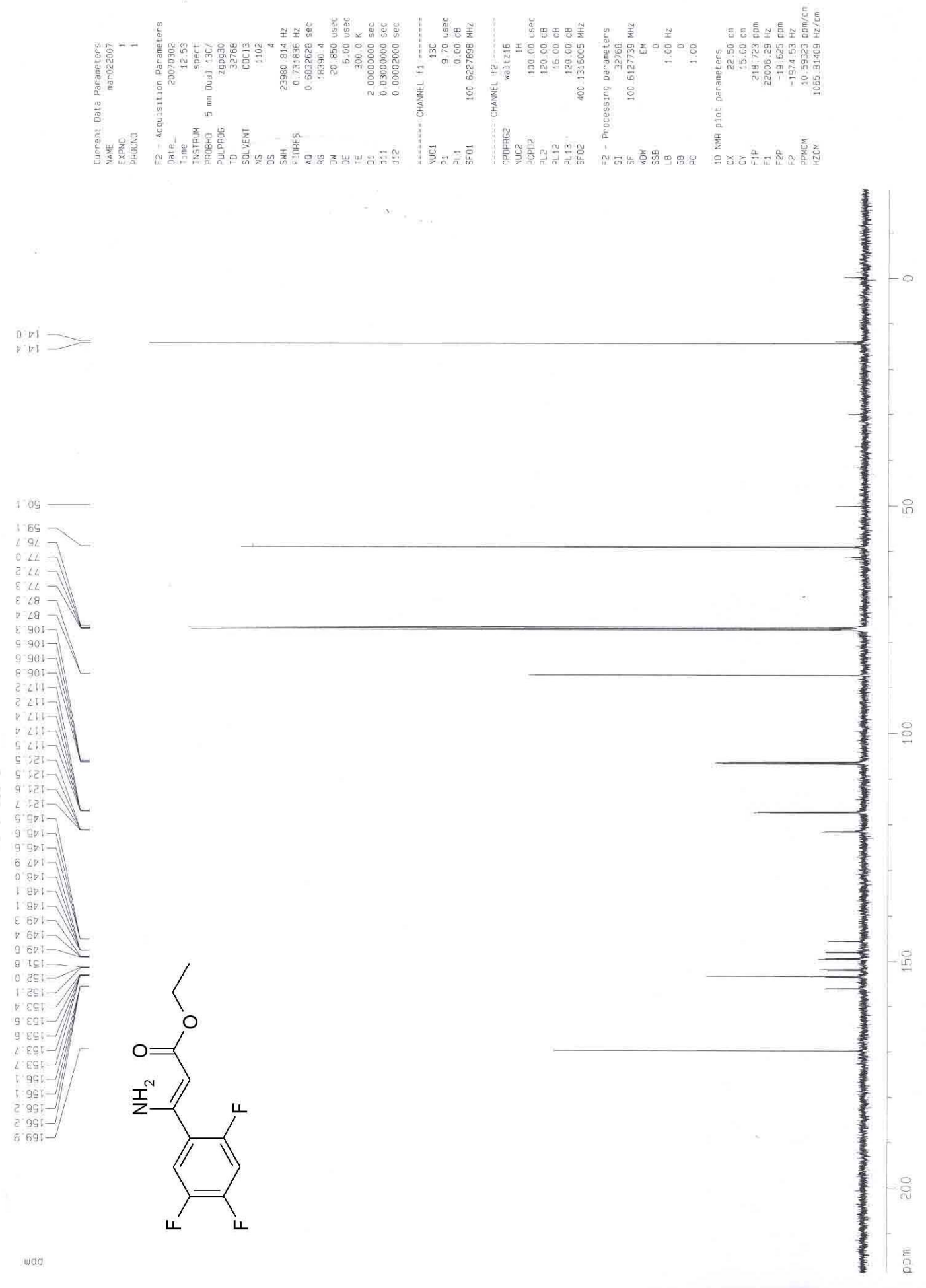
9. ${ }^{1} \mathrm{H}$ and ${ }^{13} \mathrm{C}$ spectra of $3 \mathrm{i}$

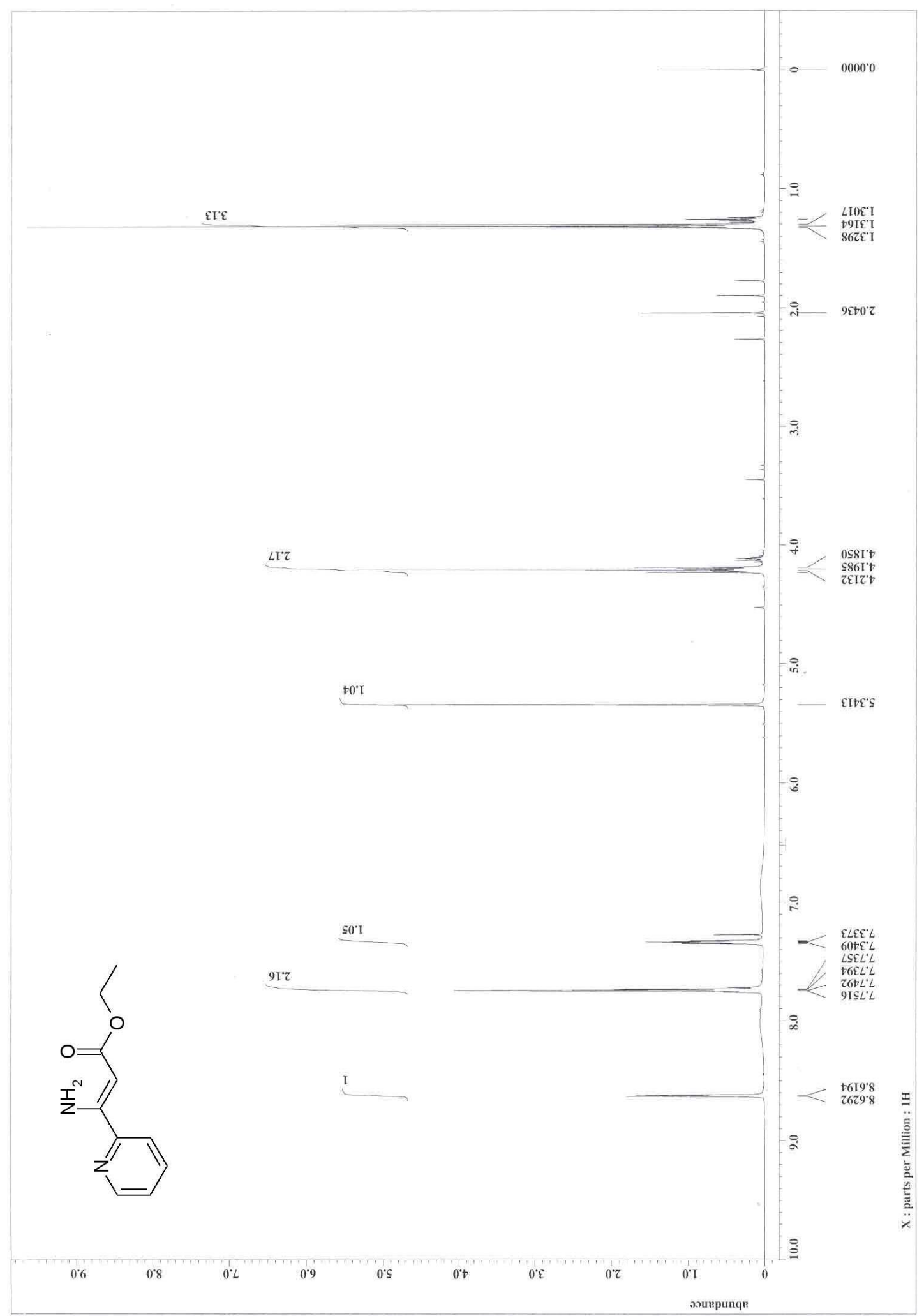




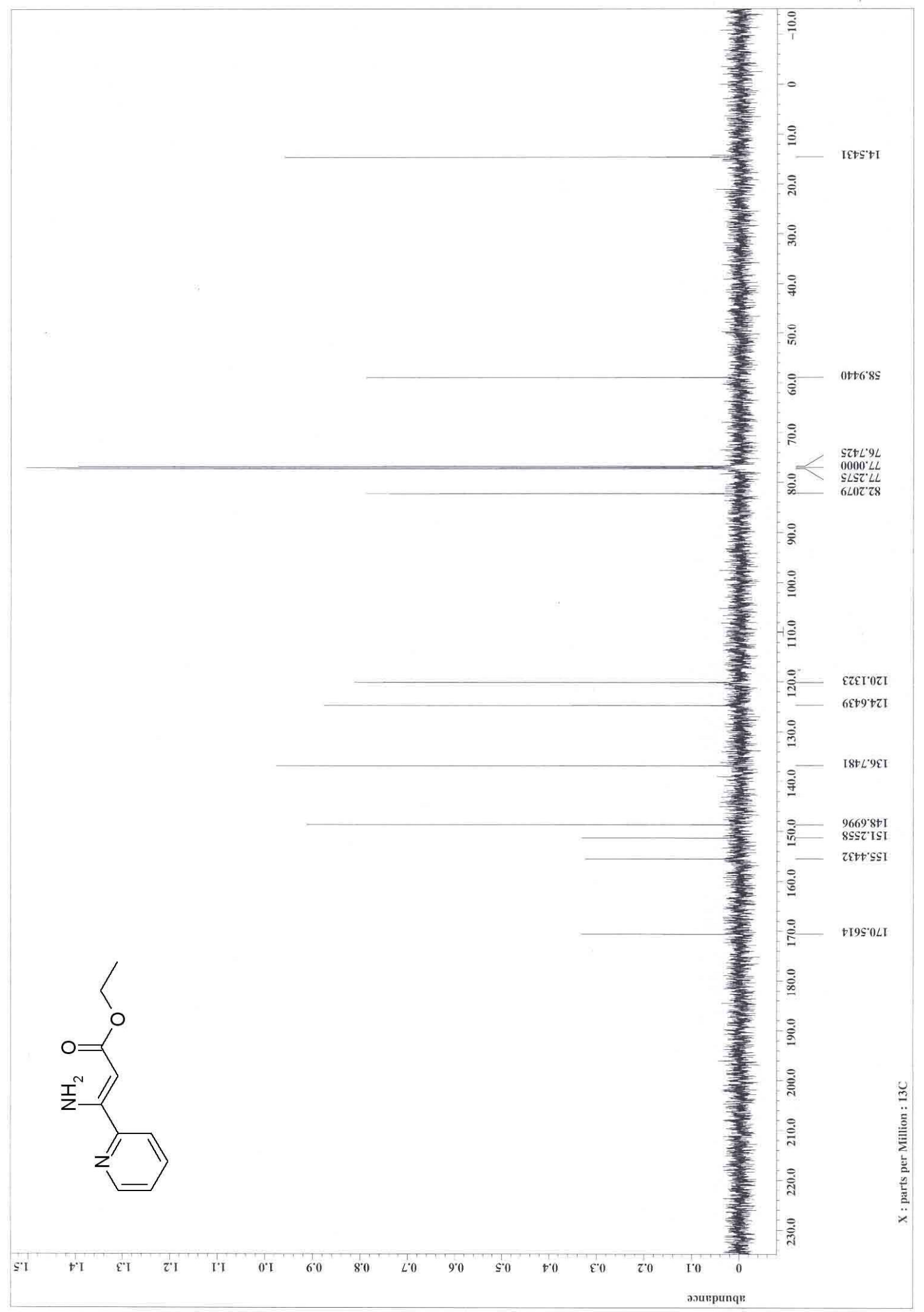


10. ${ }^{1} \mathrm{H}$ and ${ }^{13} \mathrm{C}$ spectra of $3 \mathrm{j}$

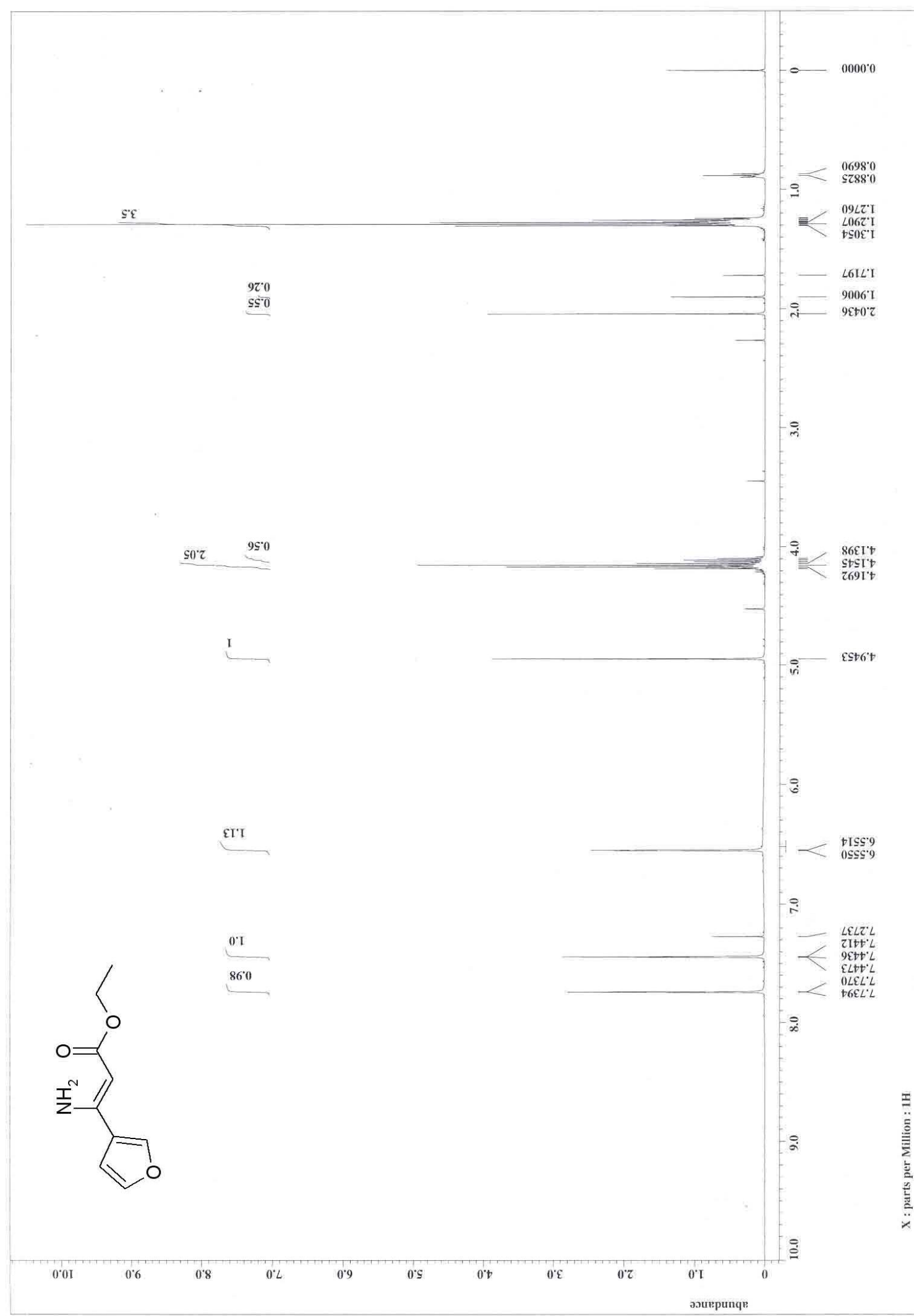




$$
1
$$


11. ${ }^{1} \mathrm{H}$ and ${ }^{13} \mathrm{C}$ spectra of $3 \mathrm{k}$

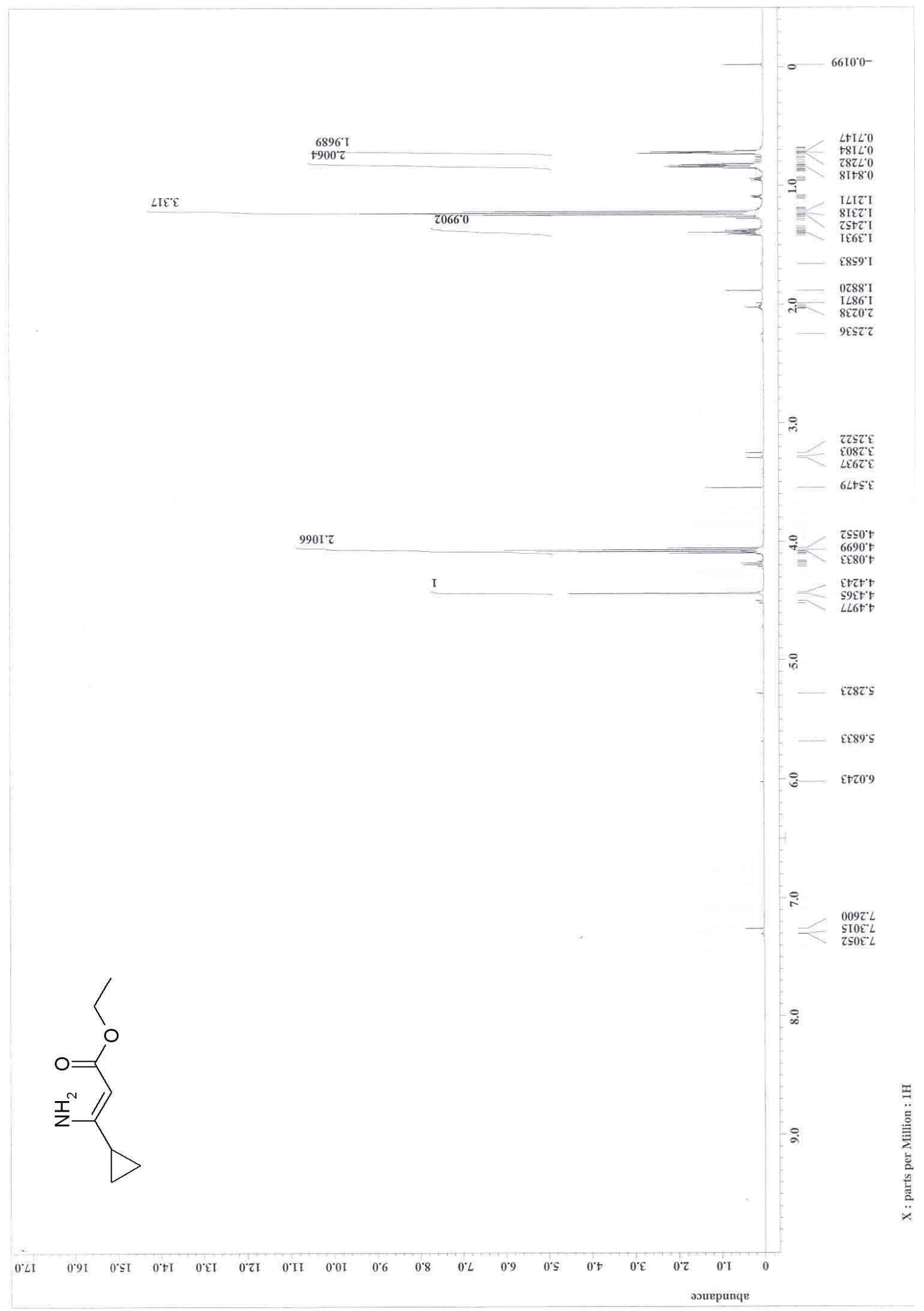




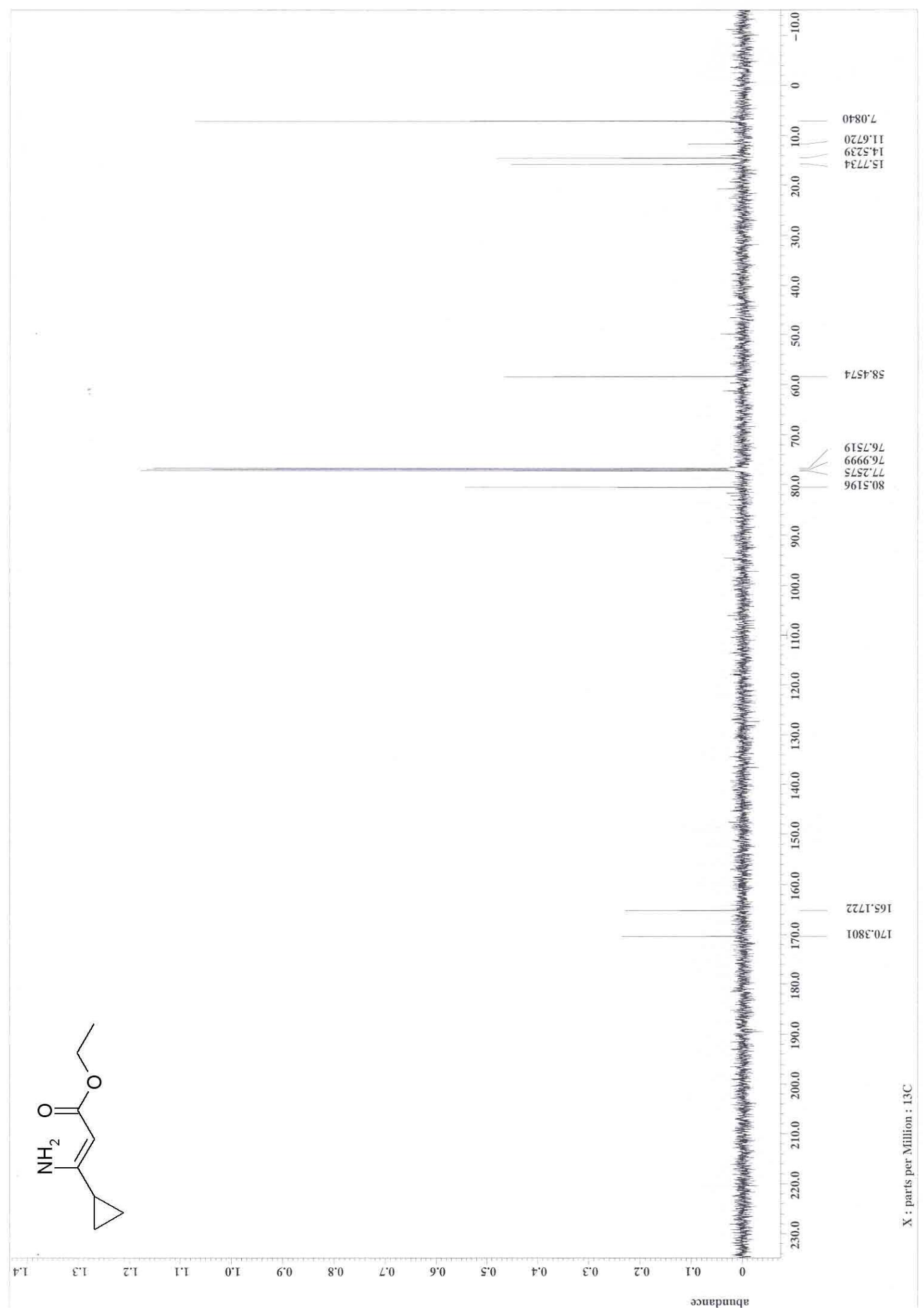

